This item is the archived peer-reviewed author-version of:

\title{
A sudden flare-up of a quiescent oral lichen planus : methylisothiazolinone as the prime suspect?
}

\section{Reference:}

Aerts Olivier, Meert Hans, Janssens Sighile, Sprengers Melissa, Chapelle Karen, Bensch Luc, Apers Sandra, Lambert Julien.- A sudden flare-up of a quiescent oral lichen planus : methylisothiazolinone as the prime suspect?

Contact dermatitis - ISSN 0105-1873 - 72:3(2015), p. 186-189

Full text (Publishers DOI): http://dx.doi.org/doi:10.1111/cod.12343

To cite this reference: http://hdl.handle.net/10067/1248670151162165141 


\section{A sudden flare-up of a quiescent oral lichen planus: methylisothiazolinone as the prime suspect?}

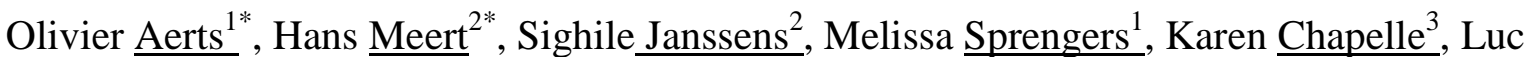
Bensch $^{4}$,Sandra Apers ${ }^{2}$, and Julien Lambert ${ }^{1}$.

*Both authors contributed equally to the manuscript.

${ }^{1}$ Department of Dermatology, University Hospital Antwerp, B-2650 Antwerp, Belgium.

${ }^{2}$ Research group Natural Products and Food - Research and Analysis (NatuRA), Department of Pharmaceutical Sciences, University of Antwerp, B-2610 Wilrijk, Antwerp, Belgium.

${ }^{3}$ Department of Stomatology, Hospital AZ Sint Maarten, B-2570 Duffel, Antwerp, Belgium.

${ }^{4}$ Special Care Dentistry Unit, University Hospital Antwerp, B-2650 Antwerp, Belgium.

\section{Correspondence:}

Dr. Olivier Aerts

Department of Dermatology

University Hospital Antwerp

Wilrijkstraat 10

B-2650 Antwerp, Belgium

Tel: +3238213273

Fax: +3238253428

E-mail: olivier.aerts@uza.be

Funding: this research was performed with financial support of the Dermatology Department of the University Hospital of Antwerp and the External Research Fund of the University of Antwerp, B-2650 Edegem, Antwerp, Belgium.

\section{Conflicts of interest: none.}

Key words: acrylates, contact allergy, dental prosthesis, dish washing liquid, high performance liquid chromatography, household detergent, koebnerization, lichen planus, methylisothiazolinone, mucosa, penetration, methyl methacrylate. 


\section{Case report}

A 61-year-old woman with a history of an asymptomatic oral lichen planus, proven by mucosal biopsy 15 years ago, was referred to our department because of oral complaints that had occurred in September 2013, shortly after the placement of a new upper dental prosthesis (consisting of a metal frame made out of a Vitallium ${ }^{\circledR}$ alloy, containing cobalt, chromium and molybdenum, and acrylate tooth material made out of polymerized methyl methacrylate (PMMA); Fig. 1). The prosthesis was used by the patient without any fixating glue. She informed us that after a few weeks the wearing of the prosthesis had resulted in intraoral swelling, vesicles and small painful, aphthous wounds accompanied by a metal taste and a dry mouth. Whenever she removed the prosthesis, the complaints settled after several hours only to recur when she tried using the prosthesis again. At the time of presentation, 6 months later, clinical examination showed an inflammatory lichenoid aspect of the buccal mucosa (Fig. 1), particularly those parts in contact with the prosthesis i.e. the lateral borders of the tongue, the gingiva and the upper palate. Because of the suspicion of a contact allergy patch tests were performed with the Belgian baseline series (including methylisothiazolinone (MI) 500 ppm aq. and supplemented with MI 2000 ppm aq., methylchloroisothiazolinone (MCI)/MI 200 ppm aq., benzisothiazolinone (BIT) 500 ppm pet. and octylisothiazolinone (OIT) 1000 ppm pet.), a dental series, an acrylate series (stored in the freezer), a bakery series, all by Chemotechnique ® (Chemotechnique Diagnostics, Vellinge, Sweden) and (ingredients of) her personal dental hygiene products. Readings were performed according to International Contact Dermatitis Research Group (ICDRG) guidelines and showed no reactions at D2, but at D4 1+ reactions were recorded for the mixtures of MCI/MI $100 \mathrm{ppm}$ aq. and 200 ppm aq. as well as for MI 500 ppm aq. and 2000 ppm aq. (Fig. 3). There were no reactions to BIT or to OIT, and no late reactions ( $\geq$ D7) were observed. Interestingly, the patient had never experienced any contact dermatitis due to skin contact with cosmetics, household detergents, paints or glues. However, she informed us that she had taken the advice of her dentist to clean her prosthesis twice weekly by soaking it, usually for 15 to 30 minutes, but sometimes up to 1 hour, into a jar filled with water to which a highly concentrated dish washing liquid (Dreft $®$ [Fairy $\left.{ }^{\circledR}\right]$, Procter \& Gamble ${ }^{\circledR}$, Strombeek-Bever, Belgium) was added. The label of the detergent mentioned the presence of MI and to verify this a chemical analysis was performed at the Research group Natural Products and Food - Research and Analysis (NatuRA) of the Antwerp University. Briefly, an amount of $1 \mathrm{~g}$ of the detergent was dissolved in $6 \mathrm{ml}$ methanol and placed for $30 \mathrm{~min}$ in an ultrasonic bath. Afterwards the solution was quantitatively transferred to a volumetric flask of $10.0 \mathrm{ml}$ and adjusted to volume with methanol. Prior to analysis the solution was filtered $(0.45 \mu \mathrm{m})$. The obtained solution was then analysed using highperformance liquid chromatography (HPLC) with a UV-detector. The column used was a GraceSmart RP C ${ }_{18}$-column ( $5 \mu \mathrm{m} ; 4.6$ x $250 \mathrm{~mm}$ ) (Grace Alletech ${ }^{\circledR}$, Deerfield, Illinois, United States), the mobile phase was formic acid $0.1 \%(\mathrm{v} / \mathrm{v})$ in water (solvent A)/acetonitrile (solvent B) using gradient elution, the flow rate was $1 \mathrm{ml} / \mathrm{min}$, the injected volume was $20 \mu \mathrm{l}$ and the wavelength used for the detection of MI was $274 \mathrm{~nm}$. Comparison with a standard solution of MI (obtained from Santa Cruz Biotechnology $®$, Santa Cruz, United States) showed indeed the presence of $\mathrm{MI}$ in the detergent which could be quantified at about $135 \mathrm{ppm}$ (mean of 2 independent results). No MCI, BIT or OIT were found. A spike experiment (adding a known amount of MI, MCI, BIT and OIT to the sample) confirmed the suitability of the applied method for this sample, resulting in a recovery between 97.3 to $103.1 \%$ for the 4 isothiazolinones. The patient was instructed to stop the cleansing procedure and to thoroughly clean her prosthesis by soaking it into plain water and rinsing it several times before using it again. Also, her dentist 
recommended using an alternative prosthesis-friendly cleaning method (Dentipur gel $®$, HelagoPharma GmbH, Erftstadt, Germany). Moreover, the remaining active lichenoid lesions, which showed a waxing and waning course, were treated using a topical betamethasone dipropionate $0.05 \%$ mouth paste. When contacted again after 2 weeks, she had cleaned the prosthesis 3 times which had enabled her to wear it already for a longer period of time during the day and at a second follow-up at 6 weeks she was again wearing the prosthesis continuously during the day. Besides inadequate rinsing, also possible penetration of MI into the acrylate component of the prosthesis was coined as a possible explanation. In order to verify the latter hypothesis, the following experiment was done: three small pieces of PMMA $\left(\sim 1 \mathrm{~cm}^{2}\right)$ were delivered by the dental technical laboratory which had manufactured the prosthesis: (i) one piece was soaked for 1 hour into $15 \mathrm{~mL}$ of water to which 2 drops $(\sim 0.1 \mathrm{~mL})$ of the MI-containing household detergent were added twice weekly for 4 weeks and after each procedure it was thoroughly rinsed for 2 minutes; (ii) one piece was treated the same way, but with MI 500 ppm aq. (Chemotechnique diagnostics ${ }^{\circledR}$, Chemotechnique, Vellinge Sweden); (iii) one piece was treated with only water and served as a control. The patient agreed to be tested with all three pieces of PMMA placed on the upper arm for 48 hours. Readings at D2 and D4, unfortunately, remained negative. An additional effort to demonstrate the penetration of MI was undertaken by soaking a similar PMMA-based prosthesis into a jar containing $150 \mathrm{~mL}$ of water to which 2 drops of the same detergent were added. After 2 days, it was thoroughly rinsed and cleaned in fresh water using an ultrasonic bath for 5 minutes. The residual water, concentrated after evaporation, was then analysed, using the aforementioned HPLC-method, showing, however, no MI.

\section{Discussion and conclusion}

Dental prostheses can be the cause of oral contact allergy due to metals or, more rarely, due to the acrylates used to manufacture them (1). In our case MI was considered the cause of a sudden flare-up of a latent oral lichen planus due to its presence in a household detergent that was used to clean a prosthesis. Besides cosmetics and paints, household detergents have been pointed out as important allergen sources of MI, the latter mainly in relation to hand dermatitis (2). Since the labelling of household detergents is mandatory, but not always correct (3), and because we did not dispose of any information on the range of use concentrations of MI in the particular dish washing liquid, we verified its presence and concentration by means of HPLC-UV which actually showed a high content of MI ( $\pm 135 \mathrm{ppm})$. Although the use of a household detergent to clean a dental prosthesis might seem erroneous, some dentists advice this, as can also be witnessed when consulting some commercial dental care websites (4).

We hypothesize that mainly inadequate rinsing of the prosthesis, with remaining of the film forming detergent and MI herein present, might have triggered the reaction in our patient. Alternatively, MI might have penetrated the porous structure of the acrylate-component of the prosthesis, possibly even accumulating in it with repeated soaking, since the acrylate part of such dental prostheses, usually made out of PMMA, is not a solid material, but a porous one with the degree of porosity depending on several factors (e.g. the amount of residual monomers) which might lead to the harboring of micro-organisms (e.g. Candida albicans), but also to the retention of fluids (5). Interestingly, it was recently published that MI might penetrate through natural rubber latex gloves (6), and a similar observation was reported by Fukunaga et al. who described a case where OIT, the isothiazolinone structurally most related to MI, penetrated from the inner gel layer of a mattress, through a middle polyethylene layer, to the outer textile layer, causing 
contact dermatitis (7). Our efforts to demonstrate such penetration of MI into the dental acrylate material were unsuccessful and several reasons might explain this: (i) we could only test small pieces of acrylate material $\left(\sim 1 \mathrm{~cm}^{2}\right)$ with the absolute MI-content being possibly too low to elicit reactions when tested ' as is' onto the skin, (ii) a similar, but not identical, PMMA-based prosthesis was used for the experiment, exhibiting possible differences with regard to polymerization status and amount of residual monomers, hence influencing the degree of porosity of the prosthesis; moreover, the soaking of this prosthesis in the MI-based solution for only 2 days might have been too short in order to ensure enough accumulation of MI inside the prosthesis for the described HPLC-method to detect its presence.

Our patient never experienced any hand eczema or other dermatitis after the use of MI- or MCI/MI-containing cosmetics or detergents, which, after careful examination of her personal care and household products, only consisted of rinse-off products. Moreover, the patch test reactions in our patient were all of a mild 1+ intensity and therefore rinse-off cosmetics or detergents containing MI might not necessarily produce clinical symptoms in such low-grade sensitized patients, as has also been described for MCI/MI-containing rinse-off products in MCI/MI-allergic patients (8). Alternatively, our patient might represent a case of contact allergy to MI with clinical relevance only at the mucosal level without, however, contact dermatitis of the skin. It is of note that MI can also be present in sexual lubricants which are used on to the anogenital mucosa and cause MI-related genital dermatitis (2).

In conclusion, we report a case where MI, present in a household detergent used to clean a dental prosthesis, might have caused primary mucosal sensitization, resulting in koebnerization and thus leading to a flare-up of a long-lasting, but hitherto inactive, oral lichen planus. Apart from its toxicity, also its allergenic potential makes it clear that the use of MI at the mucosal level should be avoided. In the future, one might focus on the potential of the isothiazolinones to penetrate solid materials such as rubbers, plastics and possibly even acrylates, the latter which, however, remains to be proven.

\section{Acknowledgment}

We are grateful to prof. An Goossens for discussing this case with us.

\section{References}

1. Khamaysi Z, Bergman R, Weltfriend S. Positive patch test reactions to allergens of the dental series and the relation to the clinical presentations. Contact Dermatitis 2006: 55: 216-218.

2. Aerts O, Baeck M, Constandt L et al. The dramatic increase in the rate of methylisothiazolinone contact allergy in Belgium: a multicentre study. Contact Dermatitis 2014: 71: 41-8.

3. Vanneste L, Persson L, Zimerson E et al. Allergic contact dermatitis caused by methylisothiazolinone from different sources, including 'mislabelled' household wet wipes. Contact Dermatitis 2013: 69: 311-2.

4. http://perfectkunstgebit.inet-solutions.nl/Onderhoudprothese.aspx; last accessed 16 September 2014. 
5. Wolfaardt JF, Cleaton-Jones P, Fatti P. The occurrence of porosity in a heat-cured poly (methyl methacrylate) denture base resin. J Prostheth Dent 1986: 55: 393-400.

6. Espasandín-Arias M, Goossens A. Natural rubber gloves might not protect against skin penetration of methylisothiazolinone. Contact Dermatitis 2014: 70: 249-51.

7. Fukunaga A, Nishiyama S, Shimizu $\mathrm{H}$ et al. Non-occupational allergic contact dermatitis from 2-N-octyl-4-isothiazolin-3-one in a Japanese mattress gel-sheet used for cooling. Contact Dermatitis 2010: 62: 317-318.

8. Fewings J, Menné T. An update of the risk assessment for methylchloroisothiazolinone/methylisothiazolinone (MCI/MI) with focus on rinse-off products. Contact Dermatitis 1999: 41: 1-13. 


\section{Figures}

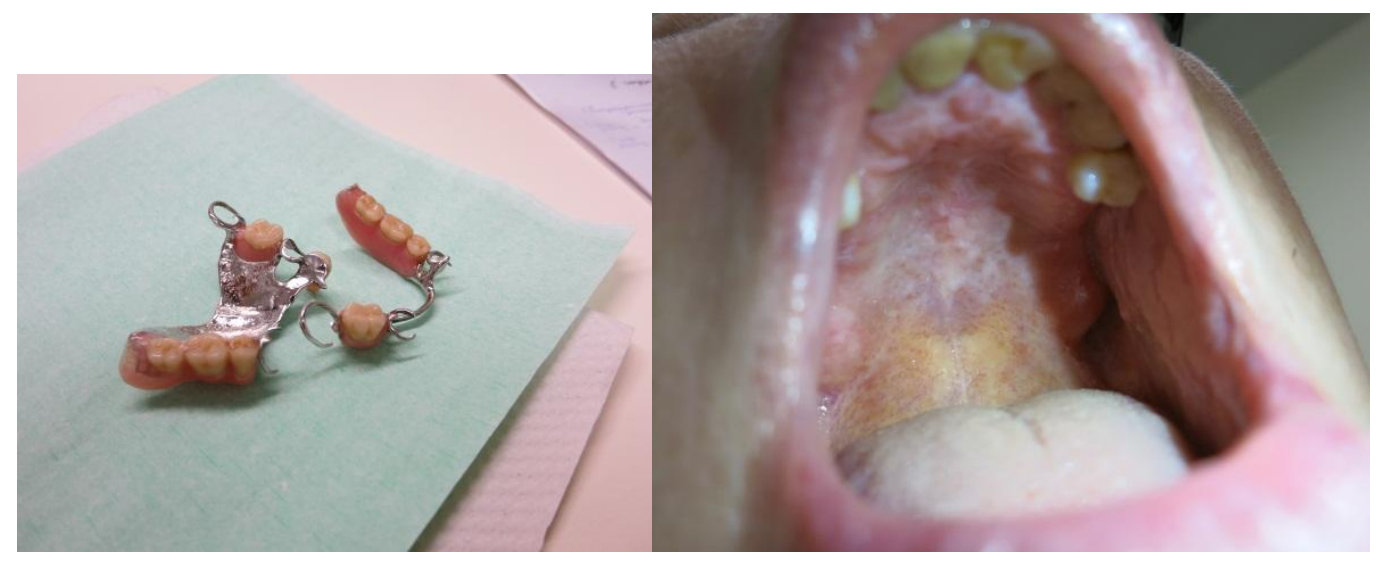

Fig. 1. A dental prosthesis containing cobalt, chromium, molybdenum and polymerized methyl methacrylate producing lichenoid lesions of the palate, gingiva and lateral borders of the tongue.

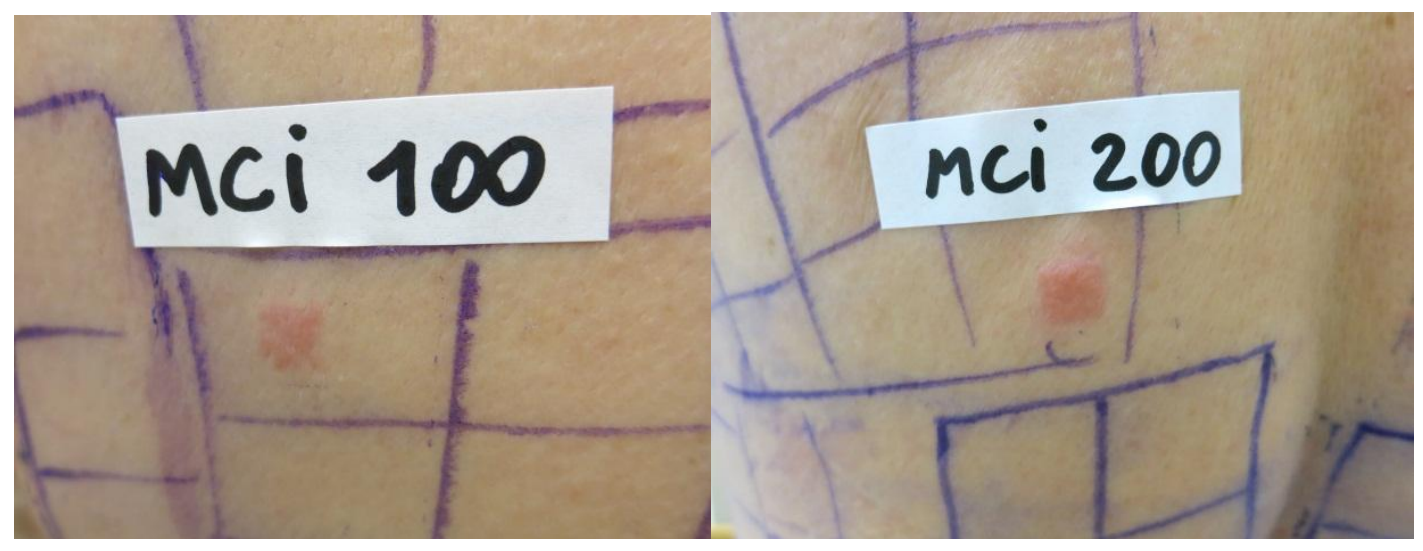

Fig. 2. MCI/MI 100 ppm aq. and 200 ppm aq. producing 1+ reactions at D4.

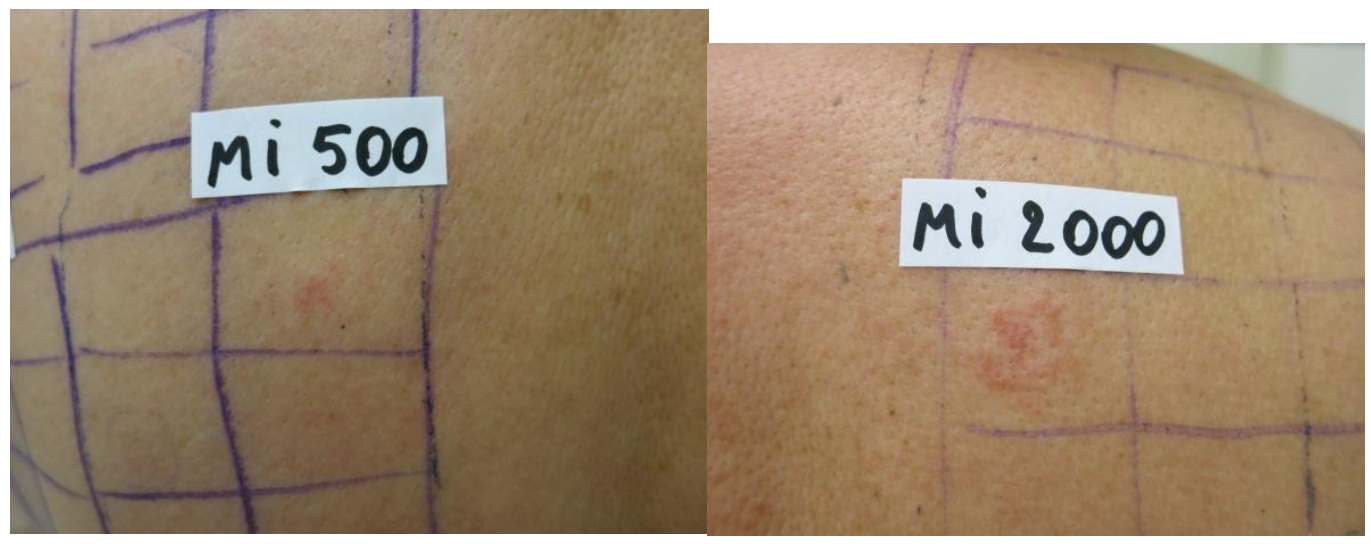

Fig. 3. MI 500 ppm aq. and 2000 ppm aq. producing 1+ reactions at D4. 